\title{
The Accuracy of Topographical Methods in Determining Central Sulcus: A Statistical Correlation Between Modern Imaging Data and These Historical Predications
}

\author{
Amy Sun ${ }^{1}$, Lewis C. Hou ${ }^{2}$, Samuel H. Cheshier ${ }^{3}$, Mark Sedrak ${ }^{4}$, Victor Tse ${ }^{4}$ \\ 1. Medical school, NYU 2. Department of Neurosurgery, Kaiser Permanente, Redwood City, California \\ 94063 3. Department of Neurosurgery and Neurology, Stanford University School of Medicine \& Lucile \\ Packard Children's Hospital 4. Department of Neurosurgery, Kaiser Permanente, Redwood City, \\ California 94063
}

$\square$ Corresponding author: Victor Tse, tsevictor@gmail.com

Disclosures can be found in Additional Information at the end of the article

\section{Abstract}

A good working knowledge of surgical anatomy has helped surgeons for centuries to choreograph operations, anticipate dangers, formulate appropriate surgical corridors, and create exit strategy. This paper reiterates the development of the topographical location of the central gyrus, the methodologies and their contributors throughout the last two centuries, and the accuracy of their methods.

Categories: Medical Education, General Surgery, Neurosurgery

Keywords: intraoperative navigation, surgical anatomy, brain surgery

\section{Introduction}

With the introduction of intraoperative navigation systems, neurosurgeons are able to optimize the surgical corridors to various regions of interest while minimizing the size of incision and craniotomy, in addition to tissue destruction [1]. Despite the advancements in intraoperative guidance technologies, familiarity with important surface anatomical landmarks continues to be indispensable knowledge that every neurosurgical trainee must develop [2]. Use of surface anatomical landmarks to localize intracranial structures are used in bedside procedures, such as in the use of Kocher's point to mark the insertion site for a ventriculostomy. Intraoperatively, landmarks such as the anatomic keyhole and coronal suture are used during pterional and bicoronal craniotomies and astrion for retromastoid approaches, respectively [3-6].

Received 03/17/2014 Review began 03/18/2014 Review ended 06/15/2014 Published 06/29/2014

\section{C) Copyright 2014}

Sun et al. This is an open access article distributed under the terms of the Creative Commons Attribution License CC-BY 3.0., which permits unrestricted use, distribution, and reproduction in any medium, provided the original author and source are credited.
Furthermore, in cases where navigation systems are not available or when the systems malfunction, surface landmarks are necessary for craniotomies to be carried out in an accurate and safe matter.

Traditionally, surface topographic localization methods were developed or validated using cadaveric-based studies as in the studies by Taylor and Haughton [7], Broca and Maubrace [8], and others in the late 1800s and in the early 1900s. However, the accuracy of this approach is often limited by a relatively small sample size and potentially tissue distortion secondary to formalin fixation [9]. In this study, we describe a practical and cost effective method to study the anatomy of central sulcus using 3D rendered CT images. In doing so, we verify the accuracy of five traditional methods of topographical localization of the central sulcus. 


\section{Materials And Methods}

\section{Patient selection}

High-resolution (1.25 mm thickness) head CT scans were collected and retrospectively analyzed for this study. Patient selection criteria included the following: 1) Lack of prior history of cranial surgery; 2) Absence of intracranial pathology that would result in the distortion of normal cerebral anatomy; and 3) Normal-appearing anatomical structures, without evidence of cortical atrophy. Twenty-six patients, representing 52 sides total, were included in this study. Among the patients, 18 were male and eight were female. The mean and median ages were 61.7 years and 60 years, respectively (range 23-77). The distribution of pathologies, in decreasing order, was Parkinson's dementia (73\%), essential tremor (19\%), and dystonia (8\%).

\section{Image analysis and data acquisition}

High-resolution CT images were uploaded into the StealthStation ${ }^{\circledR}$ TREON ${ }^{\circledR}$ Navigation System in standard fashion (Medtronic Inc, Minneapolis, MN). Image contrast was adjusted to optimize visualization of cerebral cortical anatomy. Concurrently, 3D reconstruction of the skulls was rendered and positioned laterally. The skull was rotated, such that the zygomatic arch was parallel to an imaginary X-axis. Direct tracings of the skull and bony landmarks, including the orbit, nasion, anatomic keyhole, external occipital protuberance, external auditory meatus, zygomatic arch, coronal and lambdoidal sutures, and mastoid process, were made onto a transparency. The precentral sulcus was determined based on its perpendicular intersection with the superior frontal sulcus over the cerebral convexity. The central sulcus was identified as the immediate sulcus posterior to the precentral sulcus. The central sulcus was further verified by the "Omega sign" and its corresponding primary motor cortex. Using the Stealth station's split windows mode, these anatomical land markers were co-registered in all three dimensions, the corresponding central sulcus was rendered, and its localization on the 3D rendered skull image was traced on to the transparency (Figure 1). Similarly, the course of the sylvian fissure was outlined. In some cases, the MRI images of the same patient were used and fused with the CT images to validate the localization. However, all anatomical landmarks and measurements were acquired from CT images only.

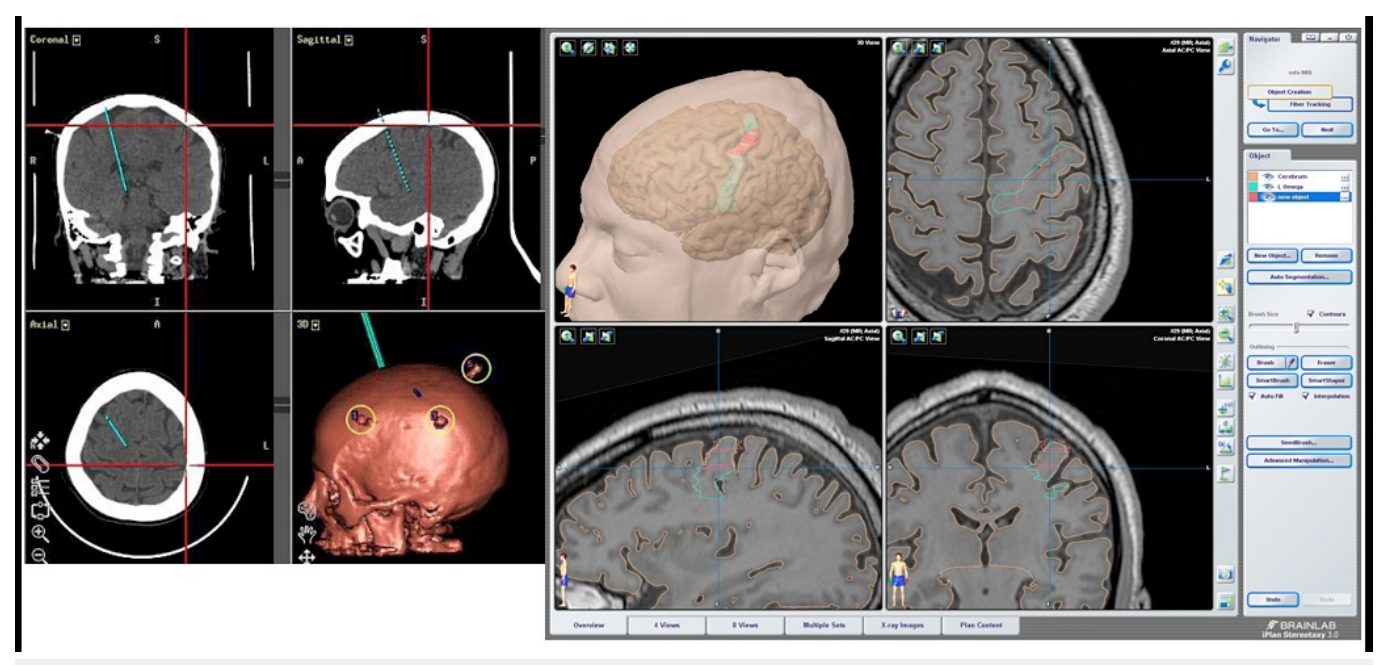

FIGURE 1: An illustration of the "real" central sulcus as is seen in modern day intra-operative navigation system after 3D rendering of a patient's MRI scan.

A screen shot captured from Stealth Image Guidance System. Note the red cross hair cursor is centered on the central sulcus. Corresponding location on the 3D rendered image is seen in the right lower screen in blue (Panel A). The location of the "Omega" is traced out and 3D rendered 
The anatomical tracings were digitized and scaled. A line extending from the superior rolandic point (SRP) and the inferior rolandic point (IRP) was drawn to represent an estimate of the "real" central sulcus anatomy based on CT imaging. The "real" IRP is then used as the coordinate $(0,0)$ to which all points of interest are referenced. Using Adobe Photoshop C2, 2006 (Adobe Systems Inc, San Jose, CA) and Mapper (c) Little Red Frog 2002-2005) software. Central sulcus, defined by each of the topographical methods (details below), were constructed (Figure 2). The SRP and IRP of each method were identified, and converted to Cartesian coordinates using software developed by the authors. Comparisons of SRP, IRP, and the slopes of the central sulci were made. Microsoft Excel software and other statistical software packages were used for statistical analysis.

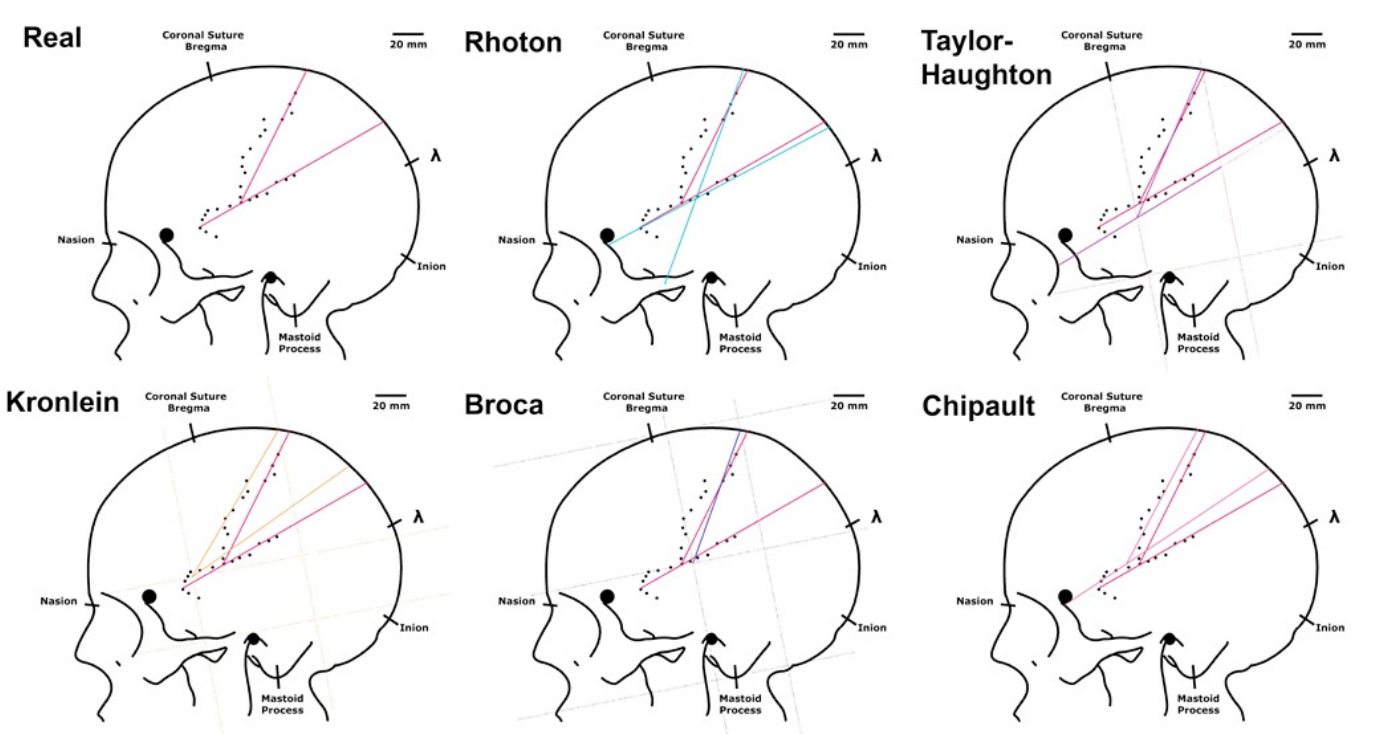

FIGURE 2: The magenta lines depict the real central sulcus (CS) and the sylvian fissure (SF). Other color lines are the predicted CS and SF according to the corresponding anatomical methods.

\section{Description of traditional topographical localization methods}

Five commonly used topographical localization methods for central sulcus were tested for their accuracy and precision in this study. These methods include: Rhoton, Taylor-Haughton, Broca, Krönlein, and Chipault.

Rhoton method: Rhoton reported the following method based on his perioperative experience 
and his collection of cadaver dissection. The distance from the nasion to inion was measured and marked at $50 \%$ and $75 \%$. The sylvian fissure was estimated by the line extending from the $75 \%$ mark to the bottom of the anatomic keyhole. The central sulcus was defined along the line from the 50\% mark to the midpoint of the zygomatic arch; the SRP, therefore, was defined as the 50\% mark; and the IRP was defined as the intersection of the two lines (Figure 2, upper middle panel) [10].

Taylor-Haughton method: This method was first described by Edward H. Taylor and Williams S. Haughton in 1900 [7]. It was based on roentgen photography on formalin-fixed cadavers. In this method, the distance from the nasion to inion was measured and marked at $50 \%$ and $75 \%$. The Frankfurt plane was drawn from the bottom of the orbit through the external auditory meatus. The posterior ear line was drawn from $2 \mathrm{~cm}$ past the $50 \%$ mark perpendicular to the Frankfurt plane and through the mastoid process. The condylar line was drawn perpendicular to the Frankfurt plane from the mandibular condyle. The sylvian fissure was defined along the line from the middle of the orbit to the $75 \%$ mark along the nasion to inion line, from the orbit to the posterior ear line. The central sulcus was defined from $2 \mathrm{~cm}$ past the $50 \%$ mark between the nasion and inion (superior rolandic point) to the intersection of the sylvian fissure and condyle line (inferior rolandic point) (Figure 2, left upper panel) [11].

Broca's method: In 1861, Broca published his method for defining the central sulci based on his studies of cadaver dissection [12]. The plane of Broca was drawn from the base of the upper teeth through the bottom edge of the mastoid process; the vertical auriculo-bregmatic line was drawn perpendicularly from the plane of Broca through the external auditory meatus to the bregma; horizontal line 9-10 was drawn parallel to the plane of Broca intersecting with the bregma; vertical line 1-2 was drawn $45 \mathrm{~mm}$ posterior to the auriculo-bregmatic line perpendicular to the plane of Broca; and the horizontal line 5-6 was drawn from the external angular process of the frontal bone to just below the lambda suture parallel to the plane of Broca. The central sulcus was defined from the most superior aspect of vertical line 1-2 that intersects with the skull (defining the SRP) to the junction of horizontal line 5-6 and the auriculo-bregmatic line (defining the IRP). The sylvian fissure was not defined for surgical accuracy, but was approximated along the horizontal line 5-6 (Figure 2, lower middle panel).

Krönlein's method: Krönlein was based on a set of gridlines around the orbit. A line was drawn to intersect with the interior border of the orbit and the superior border of the external auditory meatus, line A, the inferior base line. A second line, line B, the superior base line, was drawn parallel to and above the inferior base line which intersected with the upper margin of the orbital cavity. A third line was drawn perpendicular to the base lines to intersect with the middle of the zygomatic arch (anterior vertical line, line $\mathrm{C}$ ). A fourth line (the posterior vertical line, line D) was drawn perpendicular to base lines to intersect with the posterior border of the mastoid process. Another vertical line was drawn perpendicular to the baselines at the point of the condyloid process of the mandible, the middle vertical line. The rolandic line (the central fissure) was where the point of intersection of the anterior vertical line with the superior baseline was connected with the point where the posterior vertical line met the vertex (Figure 2, lower left panel) [13].

Chipault's method: This method relied on a set of measurements along the nasion and the inion. It divided the distance between the nasion and the inion into a $55 \%$ and $70 \%$ point. The line defining the sylvian fissure was drawn from the retro-orbital tubercle (approximated by the inferior border of the keyhole) to the $70 \%$ mark. This line was marked at $30 \%$ from the retroorbital tubercle. The central sulcus was defined from the 55\% mark (SRP) to the $30 \%$ mark along the line defining the sylvian fissure (IRP). (Figure 2, Lower Right panel) [14].

\section{Statistical methods}


Several statistical methods were used to analyze the concordance and accuracy of the estimated central sulcus against the real central sulcus. Linear regression analysis was used, with the assumption that the observations of SRP or IRP were independent from each other and could not have influenced each other. The line of best fit was determined by the real SRP and IRP. The data points that scattered around the line of best fit were assumed to have followed the Gaussian distribution.

The mixed procedure method was used to evaluate the same data set. It examined the differences between the slopes of the central sulcus generated by the navigation system (real) and those generated by other methods. This was done by weighing one or even groups of methods against others. This analysis assumed there was a common denominator within all these methods, but allowed for differences within these methods. It performed hierarchical linear analyses and computated for covariance parameter estimates, fit statistic, and solution for fixed effects [15-17].

\section{Results}

The SRP and IRP were defined in all five of the above historical methods to determine the position of the central sulcus. The derivation of the SRP and IRP determined using various methods in comparison to the true radiographic points is illustrated in Figures $3 A$, 3B. The slope of variance, as drawn from the SRP and the IRP, is shown in Figure 3C. Numerically, the variance between the true SRP and IRP can be expressed as a pair coordinate. The values of these paired coordinates are depicted in Table 1.
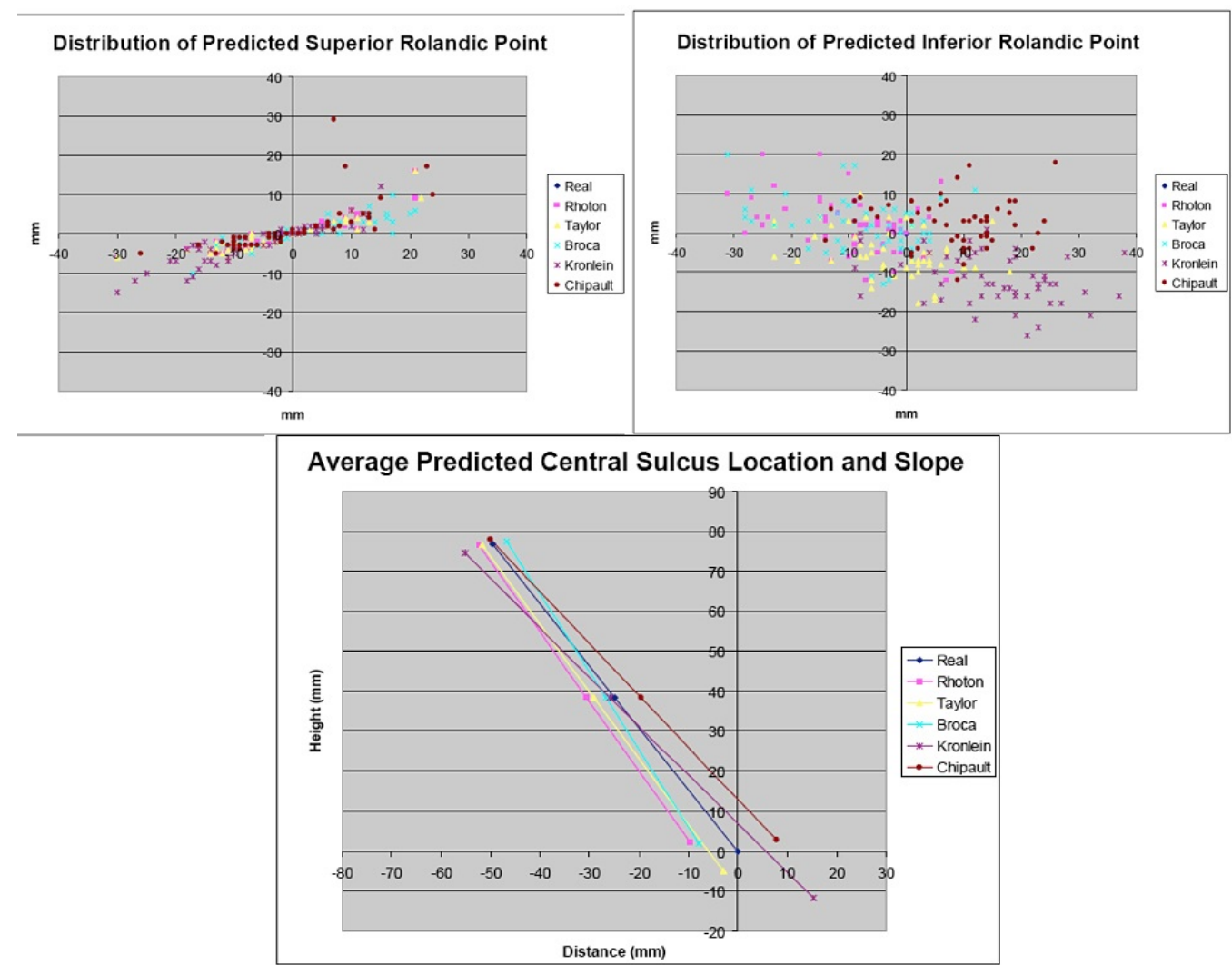

FIGURE 3: The Scatter Plot of the Predicted Superior and Inferior Rolandic Point using the Real Rolandic Point as the zero reference $(0,0)$ (Panel A and B).

The trigonometrical projections of the average predicted central sulcus determined from 


\section{Cureus}

various anatomical methods were plotted against the real central sulcus (Panel C).

\begin{tabular}{|c|c|c|c|c|c|c|c|c|c|c|}
\hline & $\begin{array}{l}\text { SRP } \\
X(\mathrm{~mm})\end{array}$ & std(MM) & $\begin{array}{l}\text { SRP } \\
Y(m m)\end{array}$ & std(mm) & $\begin{array}{l}\text { IRP } \\
\text { X(MM) }\end{array}$ & std(mm) & $\begin{array}{l}\text { IRP Y } \\
\text { (MM) }\end{array}$ & std(mm) & Slope(deg) & $\begin{array}{l}\text { std( } \\
\text { deg) }\end{array}$ \\
\hline Rhoton & -2.5 & 9.9 & +0.4 & 3.9 & -9.7 & 9.9 & +2.2 & 7.2 & +9.5 & 20.2 \\
\hline $\begin{array}{l}\text { Taylor- } \\
\text { Haughton }\end{array}$ & -2.1 & 9.9 & -0.5 & 3.8 & -2.8 & 9.2 & -5.0 & 6.0 & +6.1 & 22.1 \\
\hline Broca & +2.8 & 9.1 & +0.7 & 3.3 & -7.8 & 9.9 & +2.0 & 6.7 & +17.6 & 21.0 \\
\hline Krönlein & -5.4 & 11.1 & -2.1 & 5.1 & +15.3 & 11.0 & -11.7 & 6.3 & -17.1 & 19.3 \\
\hline Chipault & -0.3 & 9.9 & +1.2 & 6.0 & +7.8 & 9.4 & +2.8 & 6.0 & -14.0 & 19.1 \\
\hline
\end{tabular}

TABLE 1: The variance of SRP and IRP obtained from various methods were tabulated; the hypothetical line delineating the central sulcus was then computed.

The slopes simulated the deviation from the anatomical true central sulcus using various methods.

For the SRP, the average distance from the "true" SRP, as estimated by Rhoton's method, was 2.5 $+/-9.9 \mathrm{~mm}$ posterior, and $0.4+/-3.9 \mathrm{~mm}$ superior. It was $2.1+/-9.9 \mathrm{~mm}$ posterior, and $5.0+/-3.8$ $\mathrm{mm}$ inferior when using the method described by Taylor-Haughton, and when using the method described by Broca, it was 2.8+/-9.1 mm anterior and 0.7+/-3.3 mm superior. Using Krönlein's method, it was 5.4+/-11.1 mm posterior and 2.1+/-5.1 mm inferior. Chipault's method was $0.3+/-9.9 \mathrm{~mm}$ posterior and $1.2+/-6 \mathrm{~mm}$ superior.

The average distance from the "true" IRP to the IRP estimated by Rhoton's method was $9.7+/-$ $9.9 \mathrm{~mm}$ posterior, and $2.2+/-7.2 \mathrm{~mm}$ superior. Taylor-Haughton's estimation of the IRP was $2.8+/-9.2 \mathrm{~mm}$ posterior, and 5.0+/-7.1 $\mathrm{mm}$ inferior. Broca's was 7.8+/-9.9 mm posterior and 2.0+/-6.7 mm superior. Krönlein's was 15.3+/-11.0 mm anterior and 11.7+/-6.2 mm inferior. Chipault's was 7.8+/-9.4 mm anterior and 2.8+/-6 mm superior.

The trigonometric projection of the central sulcus using the SRP and IRP, as determined by Rhoton, gave a hypothetical line which was $9.5+/-20.2$ degrees clockwise from the true central sulcus; likewise, Taylor-Haughton yielded a line that was $6.1+/-22.1$ degrees clockwise from the central sulcus; Broca: 17.6+/-21 degrees clockwise; Krönlein: 17.1+/-19.3 degrees counterclockwise; and Chipault: 14.0+/-19.1 degrees counterclockwise.

In order to compare these five historical methods, several statistical methods, including the mix effect procedure analysis, were used to analyze the concordance and the accuracy of their estimation of the central sulcus (Table 2). 


\section{Cureus}

\begin{tabular}{|c|c|c|c|}
\hline Method & Estimate Standard Error & t Value & $\operatorname{Pr}>$ ItI \\
\hline Broca & -0.3230 & -3.38 & 0.0010 \\
\hline Chipault & 0.4068 & 4.26 & $<.0001$ \\
\hline Krönlein & 0.3829 & 4.01 & 0.0001 \\
\hline Rhoton & -0.09587 & -1.00 & 0.3182 \\
\hline laylor-Haughton & -0.03630 & -0.38 & 0.1049 \\
\hline
\end{tabular}

TABLE 2: The estimated error infers to the close approximation to the anatomical central sulcus, i.e. the closer to zero, the more accurate the line is toward the central sulcus.

It seems that 1) all methods were accurate in predicting the location of the SRP;2) all methods, with exception of Krönlein's, were accurate predictions of the IRP; and 3) Rhoton and TaylorHaughton have the best estimate of the trigonometric projection of the central sulcus, with Taylor-Haughton's method having the closest approximation of them all (Figure 3) (Tables 1, 2).

When this data set was further analyzed using the "Mix procedure analysis" method, we found no statistical difference between the position of the true anatomical (from the navigation system) or the empirical central sulcus predicted by these historical methods when they were stratified by the age and genders of the cadaver, albeit the fact that there was significant more variation of the location of SRP and IRP on the right side whether than the left side of the patients' heads. Among all methods in the determination of the central sulcus empirically, Rhoton and Taylor-Haughton's methods were very similar. Interestingly, using this analytical method we could group these historical methods into three groups, Group A was comprised of Rhoton and Taylor-Haughton, Group B was Broca, and Group C was Chipault and Krönlein. Group A seemed to have the best estimation in comparison to the other two (see Table 3). 


\section{Cureus}

\begin{tabular}{|c|c|c|c|c|c|}
\hline & Nasion/inion & $\begin{array}{l}\text { Anatomical } \\
\text { Key Hole }\end{array}$ & Zyomatic Arch & Sylivan Fissure & $\begin{array}{l}\text { Other Lines: Such as } \\
\text { Mastoid Process }\end{array}$ \\
\hline Rhoton & $50 / 75$ & Bottom & Mid-point & $\begin{array}{l}\text { Determine from N/I } \\
\text { line }\end{array}$ & Not used \\
\hline $\begin{array}{l}\text { Taylor- } \\
\text { Haughton }\end{array}$ & $50 / 75$ & $\begin{array}{l}\text { Not } \\
\text { used }\end{array}$ & $\begin{array}{l}\text { Orbit } \\
\text { to EAM/Frankfurt }\end{array}$ & Determine by $N / \mathrm{I}$ line & Used mastoid process \\
\hline Broca & Not used & Not used & Not used & Not by N/I line & Used mastoid process \\
\hline Krolein & Not used & Not used & $\begin{array}{l}\text { Orbit to } \\
\text { EAM/Frankfurt }\end{array}$ & Not by N/l line & Used mastoid process \\
\hline Chipault & $55 / 70$ & Inferior border & Not used & $\begin{array}{l}\text { Not directly referred to } \\
\text { the } N / I \text { line }\end{array}$ & Not used \\
\hline
\end{tabular}

\section{TABLE 3: A description of all definable anatomical land markers which were used in various methods.}

The use of the nasion and inion and the zygomatic arch helped to improve the localization of the central sulcus; Chipault used the nasion to inion line in a slightly different way and projected his line(s) toward the zygomatic arch, which was found to be less accurate. Additionally, simpler methods were more accurate in determining the central sulcus. Surprisingly, Broca's method came close to estimate the central sulcus, despite the fact that it was derived from a completely different and complex grid system and is chronologically much older than the other four methods that were used in this study.

\section{Discussion}

The sylvian fissure is an essential anatomical landmark with which the frontal, temporal, and parietal lobes are defined. Additionally, the relationship of the fissure and the central sulcus allows for the localization of the primary sensory and motor gyri. In the past century, these anatomical landmarks guided surgeons in their formulation and execution of surgical plans. A number of past and recent neurosurgeons/neuroanatomists have derived methods to estimate the location of these anatomical landmarks using surface topographical measurements and bony features of skull. These estimations give a close approximation to the central sulcus and sylvian fissure, and have served several generations of neurosurgeons in their quest to operate on the brain. In this study, we employ an advanced image-guided navigation system to localize these structures and to validate the accuracy of the five methods used to localize the central sulcus against the skull. Three out of five of these historical methods relied on the distance between the nasion and inion. When marked out on the sagittal plan, the cranium and its inner contents can then be defined and mapped out based on how far it is away from the nasion or the inion (Table 3).

Rhoton, Taylor-Haughton, and Chipault all use the nasion and inion as their major surface anatomical landmarks from which they draw a frame to localize the central sulcus, whereas Broca and Krönlein used different surface landmarks to identify/reference cerebral anatomy. As illustrated by our statistical analysis, topographical methods based on the nasion and inion give the best approximation to define cerebral anatomy. The nasion and inion truly 
are important features in defining the supratentorium content. They are easy to locate and geometrically, a line joining these two points, defines the longest distance in comparison to all other lines and planes that have been described. Additionally, Rhoton and Taylor-Haughton's methods incorporate the zygomatic arch as part of the major anatomical features. The zygomatic arch helps to define the temporal tip, which is located at the midpoint of the arch. Importantly, both of these methods used only two intersecting lines; their simplicity made them very user friendly, adding to their accuracy in describing the central sulcus; Rhoton's method had an additional advantage: its ease to apply in a clinical setting and with a set of well definable surface landmarks make it the most reproducible by different observers. Conversely, the method employed by Chipault, although somewhat similar to Rhoton's method, but it relied on the definition of the retro-orbital tubercle, which can be difficult to reproduce. Additionally, he employed more than two intersection points, which made the estimation method more cumbersome, thus making it a less accurate method.

Rhoton, Taylor-Haughton, Chipault and Krönlein all used triangulation, but the intersecting lines were more complicated in the writing of Chipault and Krönlein. Chipault used five trajectories, whereas Krönlein used four, which made these two methods more difficult to use and made them more subject to observer error. Chronologically, the writings of Broca and Krönlein were older than the rest, possibly handicapped by the lack of any form of imaging studies to validate and possibly refine their methods.

In fact, the use of roentography and a formalin-fixed head to correlate x-ray findings and surface anatomy was pioneered by Taylor-Haughton and was subsequently employed by other radiographic anatomists. It is noteworthy that the way Broca defined the central gyrus was rather unique in using a grid system rather than triangulation. This approach might have been influenced by the traditional writing and teaching dated back to the drawing of De Vinci. Unfortunately, the complexity of the grid system made it less user-friendly and less accurate in describing the contour of the central sulcus. Although mathematically it is easier to describe a landscape by topographical mapping using a grid system, in this case, triangulation may be more useful and applicable in a clinical setting. Even with this drawback, the accuracy of Broca's method is compatible to those of Rhoton and Taylor-Haughton and slightly more in close approximation than Chipault and Krönlein.

\section{Conclusions}

In all fairness, all the aforementioned methods have been used historically to facilitate in the planning of making the surgical incision and anatomical localization. This paper is attributed to those five anatomists and surgeons cited in this study, and to acknowledge the use of some variation of these methods in intraoperative localization even to date.

\section{Additional Information}

\section{Disclosures}

Human subjects: All authors have confirmed that this study did not involve human participants or tissue. Animal subjects: All authors have confirmed that this study did not involve animal subjects or tissue. Conflicts of interest: In compliance with the ICMJE uniform disclosure form, all authors declare the following: Payment/services info: All authors have declared that no financial support was received from any organization for the submitted work. Financial relationships: All authors have declared that they have no financial relationships at present or within the previous three years with any organizations that might have an interest in the submitted work. Other relationships: All authors have declared that there are no other relationships or activities that could appear to have influenced the submitted work. 


\section{Acknowledgements}

The authors (VT, LH) would like to express their appreciation to Dr. Stephen Nutik for his reviewing this article and his constructive comments. AS and LH contributed equally in collecting data for this paper

\section{References}

1. Hsu FP, Anderson GJ, Dogan A, Finizio J, Noguchi A, Liu KC, McMenomey SO, Delashaw JB, Jr: Extended middle fossa approach: quantitative analysis of petroclival exposure and surgical freedom as a function of successive temporal bone removal by using frameless stereotaxy. J Neurosurg. 2004, 100:695-9.

2. Ribas GC, Yasuda A, Ribas EC, Nishikuni K, Rodrigues AJ, Jr: Surgical anatomy of microneurosurgical sulcal key points. Neurosurgery. 2006, 59: ONS177-210.

3. Bozbuga M, Boran BO, Sahinoglu K: Surface anatomy of the posterolateral cranium regarding the localization of the initial burr-hole for a retrosigmoid approach. Neurosurg Rev. 2006, 29:61-3.

4. Reis CV, Sankar T, Crusius M, Zabramski JM, Deshmukh P, Rhoton AL, Jr., Spetzler RF, Preul MC: Comparative study of cranial topographic procedures: Broca's legacy toward practical brain surgery. Neurosurgery. 2008, 62:294-310. 10.1227/01.neu.0000315997.50399.91

5. Ucerler H, Govsa F: Asterion as a surgical landmark for lateral cranial base approaches . J Craniomaxillofac Surg. 2006, 34:415-20.

6. Kazkayasi M, Batay F, Bademci G, Bengi O, Tekdemir I: The morphometric and cephalometric study of anterior cranial landmarks for surgery. Minim Invasive Neurosurg. 2008, 51:21-5. $10.1055 / \mathrm{s}-2007-1022541$

7. Taylor EH, Haughton WS: Some recent researches on the topography of the convolutions and fissures of the brain. Trans R Acad Med Ireland. 1900, 18:511-522.

8. Broca A, Maubrac P: Traité de Chirurgie Cérébrale (in French). Masson, Éditeurs, Paris; 1896.

9. D'Ambrosio AL, Mocco J, Hankinson TC, Bruce JN, van Loveren HR: Quantification of the frontotemporal orbitozygomatic approach using a three-dimensional visualization and modeling application. Neurosurgery. 2008, 62:251-60. 10.1227/01.neu.0000317401.38960.f6

10. Rhoton AL, Jr: The cerebrum. Neurosurgery. 2002, 51:S1-51.

11. Taylor AJ, Haughton VM, Syvertsen A, Ho KC: Taylor-Haughton line revisited. AJNR Am J Neuroradiol. 1980, 1:55-6.

12. Broca P: Sur le principe des localisations cérébrales (article in French) . Bull Soc d'AnthII. 1861, 190-204.

13. Schultze OMS, Steward GD: Atlas and textbook of topographic and applied anatomy. WB Saunders \& Company, Philadelphia and London; 1905, pg 37.

14. DaCosta JC, Spitzka EA: Anatomy, descriptive and surgical: Henry Gray; 17th. Ed . Lea \& Febiger, Philadelphia; 1908, pg 970.

15. Crone NE, Miglioretti DL, Gordon B, Lesser RP: Functional mapping of human sensorimotor cortex with electrocorticographic spectral analysis. II. Event-related synchronization in the gamma band. Brain. 1998, 121:2301-15.

16. Peugh JL, Enders CK: Using the SPSS mixed procedure to fit cross- and longitudinal multilevel models. Educational and Psychological Measurement. 2005, 65:717-741.

10.1177/0013164405278558

17. Shimizu S, Tanriover N, Rhoton AL, Jr, Yoshioka N, Fujii K: MacCarty keyhole and inferior orbital fissure in orbitozygomatic craniotomy. Neurosurgery. 2005, 57:152-9. 